\title{
SensDeploy: efficient sensor deployment strategy for real-time localization
}

\author{
Jin-Hee Lee ${ }^{1}$ and Byeong-Seok Shin ${ }^{2^{*}}$
}

\author{
*Correspondence: \\ bsshin@inha.ac.kr \\ ${ }^{2}$ Department of Computer \\ Science and Information \\ Engineering, Inha University, \\ 100 Inha-ro, Nam-gu, \\ Incheon 22212, South Korea \\ Full list of author information \\ is available at the end of the \\ article
}

\begin{abstract}
In order to estimate the location of the user, the previous studies introduced many sensor deployment methods applying the sensor network. The important issues to consider when placing the sensors are a configuration cost and detection area of a sensor network. In other words, the sensors consisting the network should be optimally deployed by taking into account the coverage and connectivity of them. In this paper, a sensor signal is modeled as the Gaussian distribution based signal points group, and signal points in overlapping region between two different sensors are classified by e-SVM (support vector machine) method as each sensor group. In addition, a trilateration technique is used for estimating the position of a moving object. At this time, we efficiently deploy the sensors with $f$-Apriori method to maintain the connectivity between the sensors as well as to apply the trilateration. The proposed method can be utilized for optimal placement of sensors if we know a detection range of one sensor. In this paper, we introduce more effective and adaptive deployment method to consist the sensor network as taking into account the cost and the situation.
\end{abstract}

Keywords: Sensor network, Sensor deployment, Localization, Machine learning

\section{Introduction}

Many sensor networks have been used in various fields such as agricultural management [1], traffic control [2], emergency navigation [3] and security surveillance [4]. In particular, wireless sensor networks (WSNs) provide a powerful and effective means for monitoring and controlling environment. A WSN is composed of a number of tiny and low-power devices such as transceivers, actuators, and oscillators [5]. In a WSN, a large number of sensors are deployed in a field of interest to collect and process data. Since sensor deployment affects the cost and detection capability of a WSN, it is one of the key topics addressed in the researches of WSNs. It is well known that effective sensor deployment methods can greatly improve the performance of a WSN in a wide range of applications, including surveillance, monitoring, robot navigation, and location tracking [6].

The studies in [7-9] have suggested sensor deployment method that aim to optimize the coverage of individual sensors in indoor environments. In this paper, we propose a sensor deployment strategy that optimally deploys sensors as well as efficiently achieves coverage and connectivity properties between sensors. In addition, it can use to localize moving objects within the sensing range of the network since it ensures the trilateration 
connectivity between sensors placed in the path for tracking the mobile object. Therefore, we propose an optimized deployment strategy of sensors through a method to classify signal points within overlapped area between sensors and a method to place sensors for tracing of the moving object. Classification method for signal area between sensors was developed based on SVM classifier and trilateration technique for estimating the position was developed based on Apriori method. Therefore, we do not use conventional methods based on geometry and we model the sensor based on the point with low computational complexity. Thus, we propose a novel method to deploy the sensor with minimum cost while maintaining the accuracy for localization compared with the previous methods.

\section{Related work}

Sensor deployment problems have been studied in a variety of fields, including machine learning, robotics, computer vision, and computational geometry [10-18]. Especially in the field of machine learning and computational geometry, researches on sensor coverage issues are actively under way. The works use mesh-based geometry approaches to address the coverage issues in sensor deployment, such as different sensing ranges, relationships between sensors, and overlapped sensor coverage areas [19-23]. Typically, a sensor is modelled in triangular and hexagonal patterns, and signal points set of a sensor is generated by using a Voronoi diagram (or a Delaunay triangulation) to achieve full coverage and connectivity [24-26]. Our sensor deployment strategy is based on trilateration to determine the location of a mobile object, so it requires that at least three sensors shall be detected on all the places in the path. Therefore, the proposed strategy can deploy sensors as a constant distance determined according to the sensing range of sensors, as well as satisfy full connectivity between sensors in the path.

In sensor network, another important deployment issue is the classification of signal points in the overlapped area between sensors. The classifiers used in many fields are support vector machines (SVM) and Bayesian decision theory [27-30]. SVM and Bayesian decision techniques can effectively solve the quadratic programming problem when classifying a large number of data sets with the same coefficient. SVM is used to predict or classify as basically a non-probabilistic binary linear classifier. Our strategy assumes that given data (a signal point) belongs to one of two classes, and it uses the SVM classifier to produce a hyperplane that maximizes the margin between the two classes. The Bayesian decision theory is a method of classifying the data so as to minimize decision errors. In experiment, we compare the proposed method with the minimax Bayesian decision theory of the previous research [27] to minimize the maximum error when one signal data is within the overlapped area of two classes with the same distribution probability.

Most of all, overlapped sensor coverage areas should be minimized for an optimal sensor deployment. Apriori algorithm and the frequent-pattern tree (FP-tree) structure are commonly used to find the association rules between the data in the class [31, 32]. Apriori algorithm finds item datasets with high frequency included in several classes and evaluates the association between datasets as using threshold values, called support and confidence. It repeats the process until it can no longer find an associated dataset that satisfies the values. The algorithm generates the candidate dataset with high frequency in 
each step, and reduces the execution time by repeating regular progress. The proposed sensor deployment strategy uses the Apriori algorithm to yield an optimum deployment of sensors. Our method using Apriori algorithm counts support of the given data and repeatedly selects candidate sensors to eliminate according to support. In this paper, support presents the probability of belonging to one class and confidence shows the association value between the classes. In other words, they are defined according to the overlapped degree of the classes, and are calculated as frequency of signal points in the sensor. The FP-tree algorithm spends lower cost when processing input and output data by scanning only 2 times compared to previous algorithms scanning the dataset several times. In the first step, the class of the signal points with low frequency is removal candidate set because of having low degree of overlapped with different classes. In the second step, the class with highest frequency is selected from the overlapped signal points and it is stored in the FP-tree.

In both algorithms, we define the frequency that is the number of overlapped classes with one class, and arrange the sensors while repeating the process of removing the sensors with the highest frequency. However, the methods have an issue that a large amount of memory is required compared with other algorithms. In order to solve this problem, we only process the whole dataset as the number of scanning times is predetermined and the number of the candidate set is limited.

\section{Efficient location tracking strategy}

We propose efficient sensor deployment strategy in wide area. A sensor is modelled as using the strength distribution of signal based on Gaussian, and the sensors are deployed as considering connectivity and coverage between sensors for tracking of moving target in real time. Therefore, we propose methods that classifies the signal points in the overlapping area of the sensors using the $e$-SVM, and selects the sensors for trilateration using the $f$-Apriori.

\section{Signal classification using e-SVM}

The sensor signal is simulated as taking into account the characteristics of the sensor that the signal strength decreases as its distance from the signaling node increases. Figure 1a shows the sensor strength based on Gaussian distribution model, and Fig. 1b presents the sensor signal points generated as circle set. Figure 1c represents a polar coordinate model of the sensor signal. In Fig. 1c, the detection range of a sensor is represented by one circle with a radius of $r$, and one sensor is consisted of $n$ concentric circles with an offset of $r_{-}$off. In the concentric circles, each signal point is radiated at a constant distance of $\theta$ in the range $[-\pi,+\pi]$. The Eq. (1) calculates $r$ and Eq. (2) evaluates the position of one signal point in the circle.

$$
\begin{aligned}
& r=x_{1} \cos \theta+x_{2} \sin \theta \\
& \left(\begin{array}{l}
x_{1} \\
x_{2}
\end{array}\right)=\left(\begin{array}{cc}
\cos \theta & \cos \theta \\
\sin \theta & \sin \theta
\end{array}\right)\left(\begin{array}{c}
r_{-} \text {off } \\
s_{-} c_{0}
\end{array}\right)
\end{aligned}
$$

The signal reception of sensors can be blocked by the obstacle like nearby a building, so the actual sensing range of sensors is often different from the estimated one. In this paper, we simulate the sensor signal as considering three different cases where 


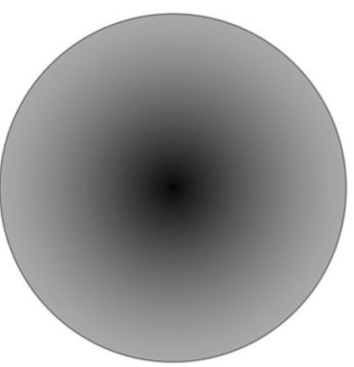

a

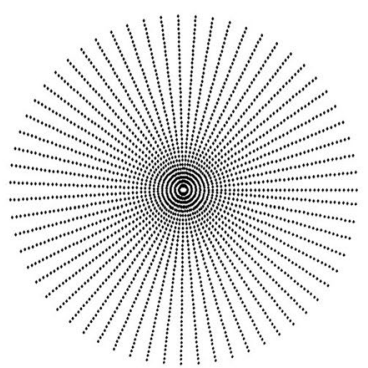

b

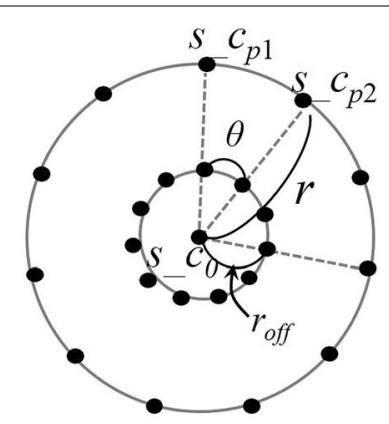

c

Fig. 1 Signal generating simulation: a sensor signal modelling based on Gaussian distribution, b point-based sensor signal simulation, and $\mathbf{c}$ signal points generating on the polar coordinate

each sensor is installed in different places of a building. As depicted in Fig. 2, this work assumes the three different cases of sensor installation in outdoor, each of which has a different degree of coverage range (i.e., 270-, 180-, and 90-degree coverages). The sensor signal propagation is modelled as these three cases. When there is more than one sensor in the vicinity, the proposed method uses SVM classifier to determine which one sensor a certain signaling point should belong to, and we compare its performance with other classifiers such as $k$-NN and Bayesian in "Experimental results and discussion".

The SVM classifier is used to find the class for a signal point included in overlapped areas between sensors. The SVM finds a hyperplane $h(x)$ which maximizes the margin. The Eq. (3) below represents Lagrange multiplier for estimating maximum margin and it is used in a general SVM-based classification model. In this paper, it is applied for generating a hyperplane to classify signal points belonged in overlapped ranges. Each signal point $x$ has a feature vector in a feature space $X$. A kernel function $K$ is denoted by a $k \times k$ matrix, and it has the characteristics of being symmetric and positive-semidefinite in this paper. Here, $k$ signal points are defined as training data $x_{i}$ with label $y_{j}$. In addition, the soft margin that determines the error tolerance is defined as $C$. In the Eq. (3), the support vector is selected as $b$ with $y_{j} h_{K}\left(x_{i}\right)=1$ for all $i$. The decision rule for classifying each signal point is shown in Eq. (4). We define it as $e$-SVM that means enhanced SVM technique.

$$
\mathrm{W}(\alpha)=\sum_{\mathrm{i}=1}^{\mathrm{k}} \alpha_{i}-\frac{1}{2} \sum_{\mathrm{i}, \mathrm{j}=1}^{\mathrm{k}} y_{i} y_{j} \alpha_{i} \alpha_{j} K\left(x_{i}, x_{j}\right)
$$

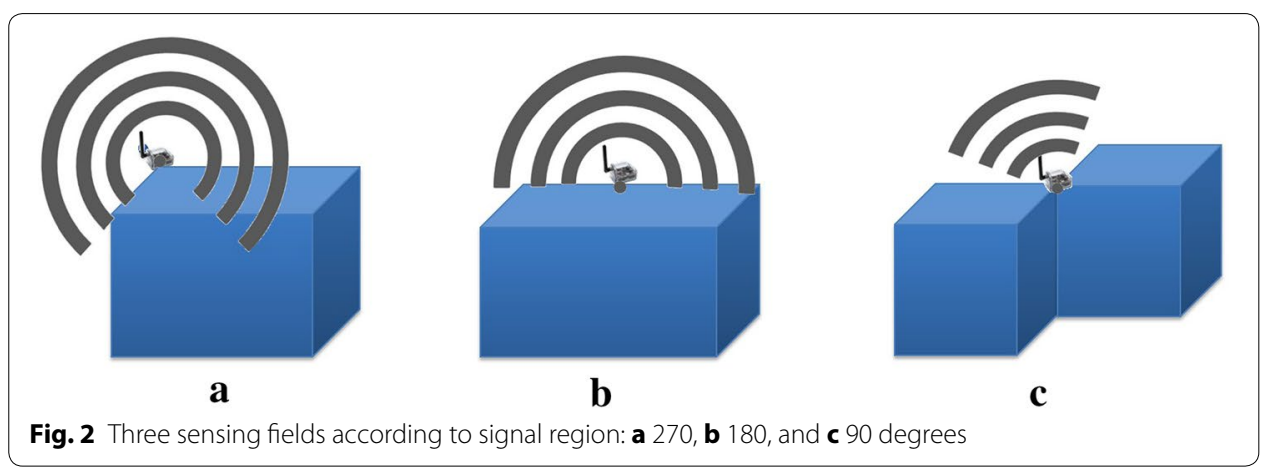


subject to

$$
\begin{aligned}
& \sum_{i=1}^{\mathrm{k}} y_{i} \alpha_{i}=0 \\
& 0 \leq \alpha_{i} \leq C, \quad i \in[1, k] \\
& \operatorname{sign}\left(h_{K}(x)\right)=1
\end{aligned}
$$

where

$$
h_{K}(x)=\sum_{i=1 \rightarrow k} y_{i} \alpha_{i} K\left(x, x_{i}\right)+b
$$

With two classes $p$ and $q$, a binary function $B$ determines a class including a signal point. This function is represented in Eq. (5).

$$
B\left(x_{i}, h_{p q}\right)= \begin{cases}1 & \text { if } h_{p q}\left(X_{i}\right)=q \\ 0 & \text { otherwise }\end{cases}
$$

\section{Sensor placement with f-Apriori}

We use Apriori algorithm for finding optimally position of the selected sensors in previous stage. Above all, in order to place the sensors, the distance between the sensors has to be determined. We define that $F$ is the area in which the sensor is placed and $I$ is the area of interest in $F$, and $S$ is the sensor set included in $F$. After the sensors are relocated at previous stage, we find the sensors satisfying the predetermined coverage and connectivity in $I$. Here, we estimate the total entropy to maximize the number of removable sensors and minimize the remaining sensors. The total entropy is the sum of the entropy of each sensor. In Eqs. (6) and (7), $\Delta n$ means the entropy for removing one sensor, and $d_{i}$ is the signal range of one sensor and $E_{i}$ is the initial entropy of the removable sensor $i$. If there are obstacles, $d_{i}$ has to be set so that overlapping coverage is minimized. The distance between neighboring sensors satisfying the equations is $r \leq d_{i}<\sqrt{ } 3 r$. In addition, it satisfies the condition for applying the trilateration technique. This is the distance to guarantee coverage and connectivity between the sensors as shown in Fig. 3.

$$
\begin{aligned}
& \max \sum_{i \in I} \Delta n \times d_{i} \\
& \min \frac{\sum_{i \in I}\left(E_{i}-\Delta n \times d_{i}\right)}{|I|}
\end{aligned}
$$

Once the coverage and connectivity of the sensors are determined, we use the sensor deployment strategy applying Apriori algorithm to find the removable sensors. When a signal point is included in the sensing range of more than two sensors (classes), we find association rules between the sensors in overlapping area. The association rules are estimated by counting the signal points in overlapping coverage generated by nearby sensors. The user-predefined minimum support value and confidence value are applied in finding the association rules. As shown in Fig. 4, Apriori algorithm produces removable 


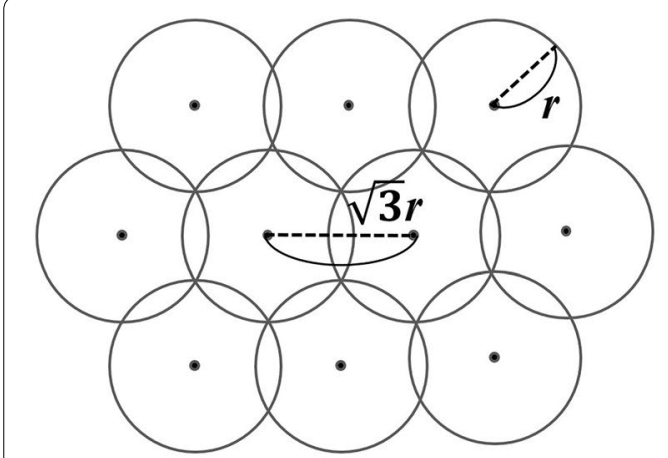

a

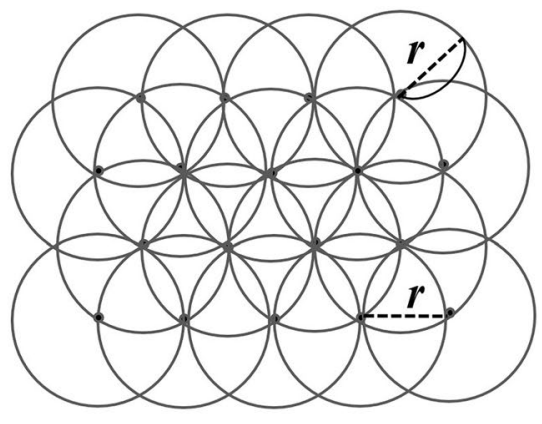

b

Fig. 3 Sensor coverage and connectivity according to sensor placement: a maximum distance and $\mathbf{b}$ minimum distance between sensors considering trilateration

candidate datasets $C$ (candidate itemset) that have high frequency in the database $D$, and extends them to longer datasets $L$ (large itemset) consisted from candidate datasets $C$. Note that the initial datasets in the database satisfy an essential prerequisite for performing trilateration, one of constraints. At each step, large itemset $L$ is generated from the candidate datasets by using a join operation. This process quickly is repeated until further large itemset $L$ are not found. Therefore, we define the method as $f$-Apriori.

\section{Experimental results and discussion}

When modeling the sensor, we experiment whether the density of the signal points according to radius ratio or angle ratio of a sensor affects the results of the proposed method. In other words, we compare and analyze the effect of parameters when use the proposed method. As shown in Fig. 5, it is an example of generating signal points in a sensor by applying the signal strength characteristic of the sensor. The sensor signal points are generated in $m$ concentric circles with the sensor signal radius $r$, radius offset $r_{o f f}$, and angle offset $\theta_{\text {off }}$ Therefore, this figure shows an example of signal points generated by a change of radius offset $r_{o f f}$ and angle offset $\theta_{o f f}$ The signal points are generated differently depending on radius offset and angle offset as shown in the figure. The figure is the result of simulation with Matlab. The simulation result shows that the finer

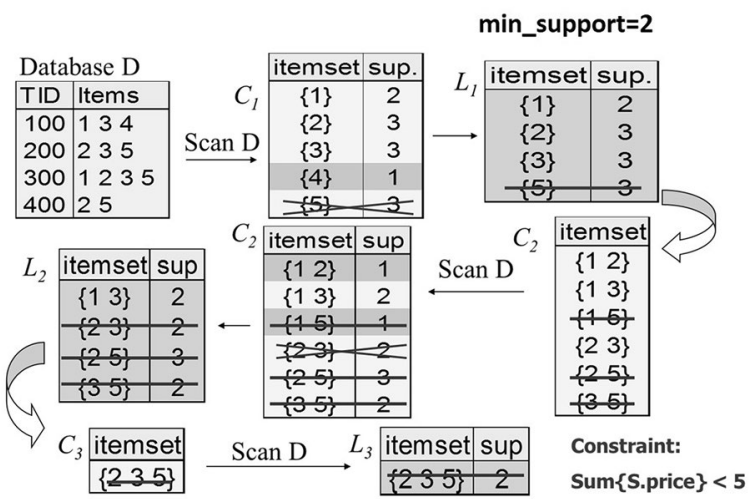

Fig. 4 Example of generating process of large itemset $L$ 
of radius offset or angle offset between signal points takes the longer time for simulation. However, there is no problem in real-time processing since this task is performed only once in the preprocessing step. In addition, the sensor modeling method proposed in this paper considers only two important parameters (radius offset and angle offset) without regard to other parameters because signal points of a sensor are generated in a circular shape based on Gaussian distribution.

In next, we experiment the change of the ratio of signal points in each sensor according to angle offset and radius offset. Then, $e$-SVM classifier is used for classifying the signal points in the overlapped area between the sensors. Each experiment evaluates the number of the signal points created by five sensors and the cases of twelve offsets. Figure 6 shows the results of signal points generated according to a change of angle offset $\theta$ off when radius offset $R \_$off is fixed at 1.0, and Fig. 7 presents the results of signal points generated according to a change of radius offset $R_{-}$off when angle offset $\theta$ off is 0.5 . As in the experimental results, each offset does not affect the ratio of the signal points in the overlapping region. In other words, the fourth sensor has the largest number of signal points because it overlaps most of the other sensors. In addition, as shown in the figure, the ratio of the signal points accumulated in five sensors is the same as that of the signal points in the range. Finally, we confirm that the offsets do not affect in the proposed method, and verify that the experimental results are the same whenever simulated at arbitrary offsets such as angle and radius. However, performance evaluation based on parameters other than the radius and angle was not performed here.

We have performed the experiment to estimate the accuracy of signal transmission in the three cases installing a sensor described in "Efficient location tracking strategy". That is, the actual receiving range of the signal is measured when the sensors have the sensing coverage of 270, 180, and 90 degrees. Figure 8 shows that the accuracy error is within a distance of 2-3.5 $\mathrm{m}$ in all three cases. However, we have not been performed experiments under a variety of sensing coverage and various environments.

In order to determine classes of signal points in overlapped area made by two sensors, we experiment the performance of the proposed $e$-SVM classifier employed in

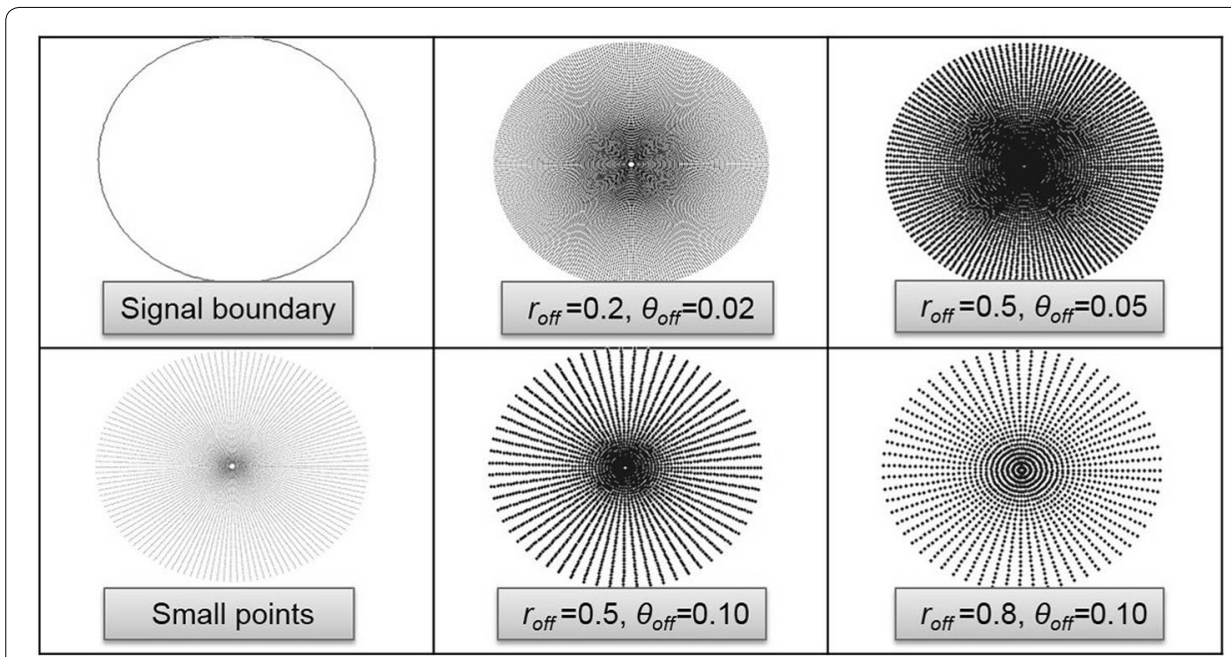

Fig. 5 Example of signal points generated by a change of radius offset $r_{\text {off }}$ and angle offset $\theta_{\text {off }}$ 


\begin{tabular}{|c|c|c|c|c|c|c|}
\hline & B_off & $1(10,10)$ & $2(-5,3)$ & $3(20,20)$ & $4(20,0)$ & $5(0,30)$ \\
\hline & 1.0 & 471 & 505 & 536 & 570 & 568 \\
\hline & 0.8 & 525 & 572 & 611 & 653 & 648 \\
\hline \multirow{5}{*}{$+t^{4}$} & 0.6 & 724 & 787 & 839 & 898 & 889 \\
\hline & 0.4 & 1047 & 1157 & 1220 & 1308 & 1296 \\
\hline & 0.2 & 2090 & 2302 & 2437 & 2615 & 2594 \\
\hline & 0.1 & 4091 & 4541 & 4806 & 5143 & 5111 \\
\hline & 0.07 & 5847 & 6488 & 6862 & 7346 & 7302 \\
\hline \multirow{3}{*}{+5} & 0.05 & 8184 & 9085 & 9609 & 10286 & 10221 \\
\hline & 0.02 & 20465 & 22718 & 24022 & 25711 & 25553 \\
\hline & 0.01 & 40833 & 45369 & 47971 & 51338 & 51023 \\
\hline \multirow[b]{2}{*}{ 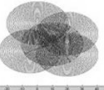 } & 0.005 & 81590 & 90685 & 95869 & 102590 & 101966 \\
\hline & 0.002 & 203935 & 226682 & 239638 & 256430 & 254875 \\
\hline
\end{tabular}

Fig. 6 Example the ratio of signal points according to angle offset $\theta$ _off

\begin{tabular}{|c|c|c|c|c|c|c|}
\hline & $R \_$off & $1(10,10)$ & $2(-5,3)$ & $3(20,20)$ & $4(20,0)$ & $5(0,30)$ \\
\hline & 1.0 & 855 & 933 & 990 & 1061 & 1053 \\
\hline & 0.8 & 1060 & 1155 & 1227 & 1314 & 1305 \\
\hline & 0.6 & 1387 & 1513 & 1608 & 1724 & 1707 \\
\hline & 0.4 & 2079 & 2266 & 2409 & 2582 & 2561 \\
\hline & 0.2 & 4113 & 4488 & 4771 & 5117 & 5073 \\
\hline & 0.1 & 8183 & 8931 & 9499 & 10187 & 10097 \\
\hline & 0.07 & 11648 & 12714 & 13523 & 14499 & 14372 \\
\hline & 0.05 & 16325 & 17816 & 18953 & 20324 & 20146 \\
\hline & 0.02 & 40751 & 44475 & 47315 & 50736 & 50291 \\
\hline & 0.01 & 81459 & 88909 & 94583 & 101424 & 100534 \\
\hline & 0.005 & 162870 & 177770 & 189118 & 202798 & 201018 \\
\hline & 0.002 & 407150 & 444320 & 472701 & 506870 & 502399 \\
\hline
\end{tabular}

Fig. 7 Example the ratio of signal points according to radius offset $R \_$off

the sensor deployment strategy that is compared with the two previous classifiers, the probabilistic Bayesian classifier in [27] and the $k$-NN method in [33]. This comparison experiment is performed in two different conditions: (a) the signals are generated irrespective of the location of sensors and (b) the signals are generated as being influenced by installed location such as the three cases described in "Efficient location tracking strategy". The sensors are placed in a residential area where buildings and other structures are densely populated, and 1500 sensors are virtually deployed in simulation. Figure 9 shows the performance of the three classifiers. In Condition 1, the proposed

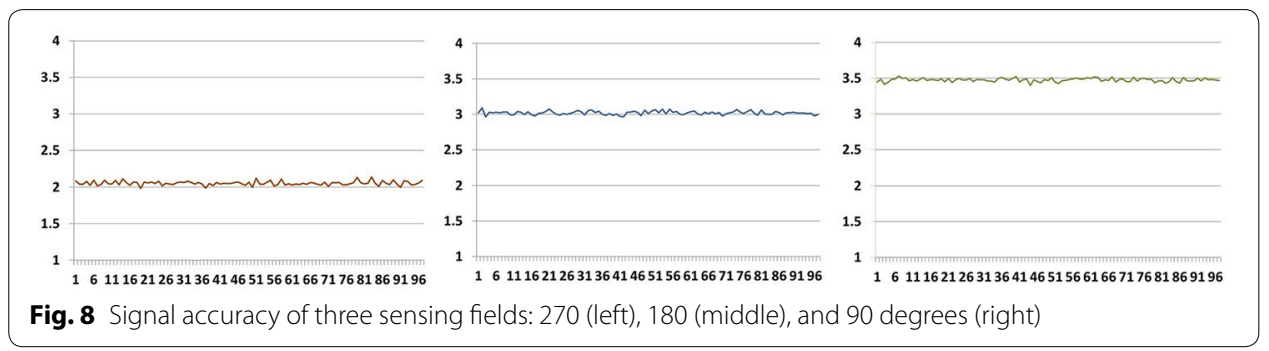


classifier can efficiently lower the cost for decreasing deployed sensors by three times in comparison with the $k$-NN classifier and 1.5 times compared with the Bayesian classifier. In Condition 2, the proposed $e$-SVM classifier reduces the number of deployed sensors by $1.8-4$ times compared to the two previous classifiers.

For optimal placement of sensors, it is important to minimize the overlapping area of sensors by making one signal point belong only to one class. In this paper, we compare the proposed $f$-Apriori method with the FP-tree method, which is similar to the proposed method, to find the sensors needed for trilateration. In other words, FP-tree is used to find removable sensors with frequent patterns through association and correlation analysis between sensors. As shown in Fig. 10, the $f$-Apriori method reduces the cost about 50\% less than the FP-tree method in both Condition 1 and 2. However, we have not performed comparative experiments on geometry-based methods and compared only the performance of two methodologically similar methods. In addition, we have not been able to compare various methods because it is difficult to model other methods by considering trilateration.

Figure 11 shows an example of the experimental results using the proposed method. We classify signal points in overlapping areas using the proposed $e$-SVM classifier. As

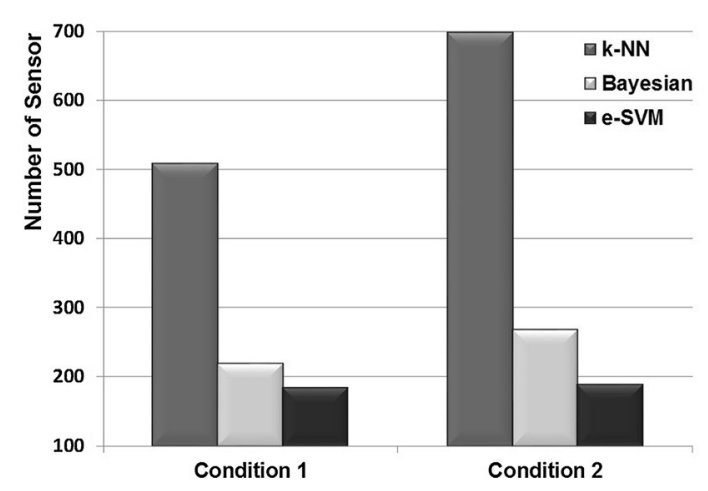

Fig. 9 The number of sensor according to each classifier

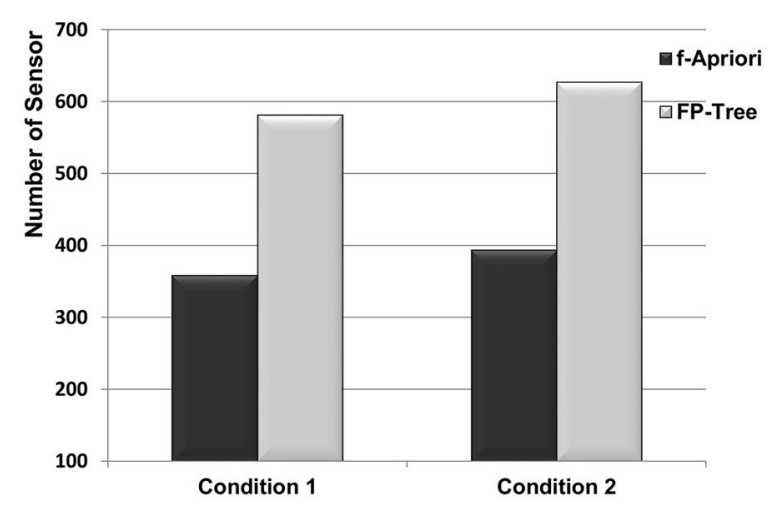

Fig. 10 The number of sensor according to each placement technique 
shown in the figure, the number of signal points voting to each sensor is accumulated to find the candidate sensors, and some of the sensors are determined as removable sensor by the proposed $f$-Apriori method. This process is repeated for each step to determine the optimum position of the sensors. The top figures show the simulation result by means of Matlab, and the bottom figures represent the signal boundary of each sensor and removable sensors (bold circle). By applying the proposed method, it is possible to optimally deploy the sensors considering the connectivity between sensors. Therefore, as shown in Fig. 11, in order to deploy the sensors, it is necessary to secure not only the distance between the sensors but also the connectivity between the sensors on the path. However, our method takes a long time when simulate for deploying multiple sensors in a large space such as outdoor environment. However, it is not a problem at all since the simulation process is performed only once at the beginning to arrange the sensor. Therefore, our method is more useful when deploying many sensors of the same type in a large space or when deploying various kinds of sensors.

\section{Conclusion}

Existing sensor deployment methods focus on maximizing the sensor field coverage in indoor environments. This paper has proposed an efficient sensor deployment strategy that covers an outdoor sensing area by deploying a minimum number of sensors while maintaining the connectivity between the deployed sensors. The proposed strategy can localize the likely position of a moving object within the sensing range of sensors via trilateration, and ensure the connectivity between transmitting and receiving sensor signal by placing the sensors along the expected motion paths of mobile object. This paper has also presented the classification of signaling points in overlapped sensor areas and the optimization of sensor deployment that enables moving objects to track anywhere in the sensing range through trilateration. In the experiments, the efficiency of the proposed $e$-SVM classifier and $f$-Apriori deployment method is demonstrated by comparing it with other algorithms.

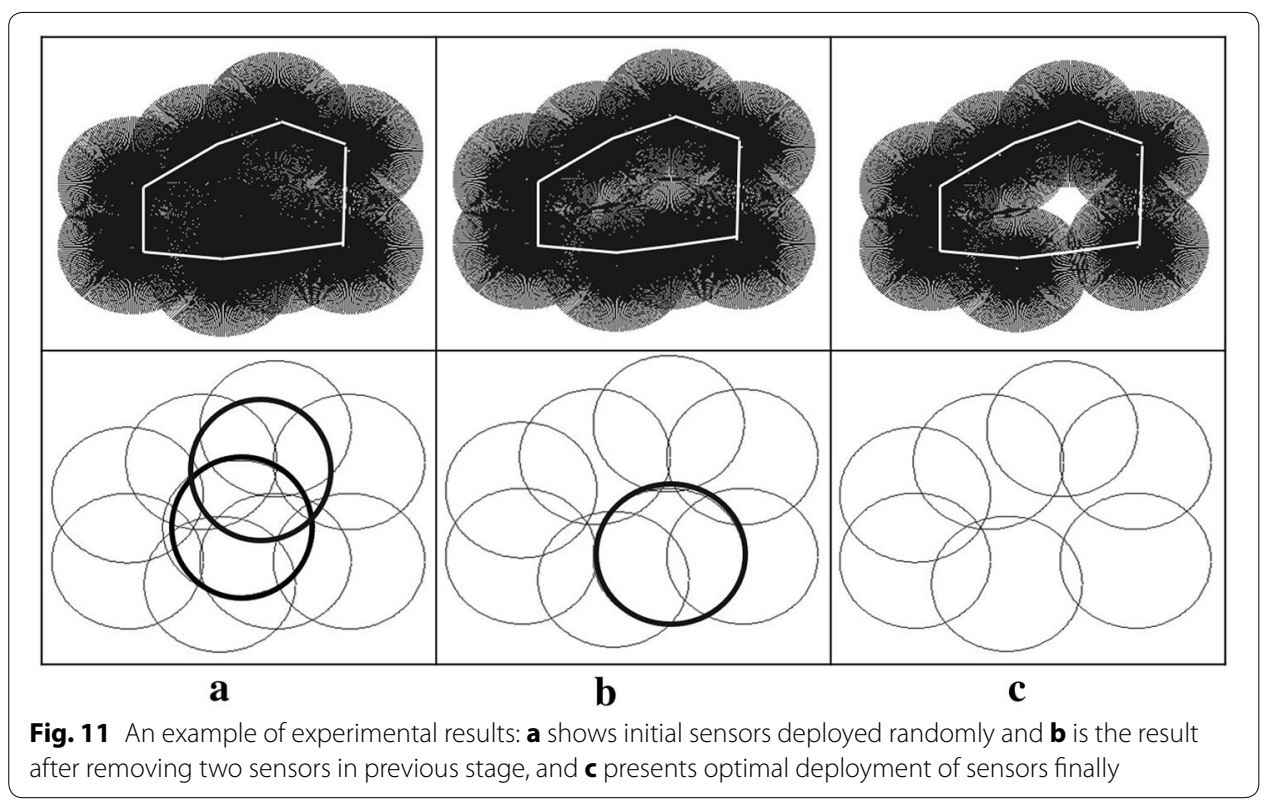




\section{Abbreviations}

SVM: support vector machine; WSN: wireless sensor networks; FP-tree: frequent pattern tree.

\section{Authors' contributions}

JHL has made substantial contributions to conception and design, or acquisition of data, or analysis and interpretation of data, as first author. BSS has been involved in drafting the manuscript or revising it critically for important intellectual content, as corresponding author. Both authors read and approved the final manuscript.

\section{Authors' information}

Jin-Hee Lee is a senior researcher in Future Automotive Technology Research Center, DGIST, Korea. She received the B.S. degree in Computer Science from Korea National Open University, and M.S. and Ph.D. degree in Computer and Information Engineering from Inha University of Korea. Her research interests include human computer interaction, wearable computer, and ubiquitous computing. Byeong-Seok Shin is a professor in the School of Computer and Information Engineering, Inha University, Korea. He received his B.S., M.S., and Ph.D. in Computer Engineering from the Seoul National University in Korea. Current research interests include human computer interaction, volume rendering, real-time graphics, and medical imaging

\section{Author details}

${ }^{1}$ Future Automotive Technology Research Center, DGIST, 333 Techno Jungang-daero, Dalseong-gun, Daegu 42988 , South Korea. ${ }^{2}$ Department of Computer Science and Information Engineering, Inha University, 100 Inha-ro, Nam-gu, Incheon 22212, South Korea.

\section{Acknowledgements}

This research was supported by next-generation information computing development program through the National Research Foundation of Korea (NRF) funded by the Ministry of Education, Science and Technology (No. 2012M3C4A7032781), and this work was supported by the DGIST R\&D Program of the Ministry of Science of Korea (16-FA-07).

\section{Competing interests}

The authors declare that they have no competing interests.

\section{Availability of data and materials}

Not applicable.

\section{Consent for publication}

Not applicable.

Ethics approval and consent to participate

Not applicable.

\section{Publisher's Note}

Springer Nature remains neutral with regard to jurisdictional claims in published maps and institutional affiliations.

Received: 15 March 2017 Accepted: 12 September 2017

Published online: 11 December 2017

\section{References}

1. Burrell J, Brooke T, Beckwith R (2004) Vineyard computing: sensor networks in agricultural production. IEEE Pervasive Comput 3(1):38-45

2. Hsieh TT (2004) Using sensor networks for highway and traffic applications. IEEE Potentials 23(2):13-16

3. Tseng YC, Pan MS, Tsai YY (2006) Wireless sensor networks for emergency navigation. Computer 39(7):55-62

4. Tseng YC, Wang YC, Cheng KY (2005) An integrated mobile surveillance and wireless sensor (iMouse) system and its detection delay analysis. In: Proceedings of ACM international symposium on modeling, analysis and simulation of wireless and mobile systems, pp 178-181

5. Akyildiz IF, Su W, Sankarasubramaniam Y, Cayirci E (2002) A survey on sensor networks. IEEE Commun Mag 40(8):102-114

6. D'Andrea R (2012) Guest editorial: a revolution in the warehouse: a retrospective on Kiva systems and the grand challenges ahead. IEEE Trans Autom Sci Eng 9(4):638-639

7. Meguerdichian S, Koushanfar F, Potkonjak M, Srivastava MB (2001) Coverage problems in wireless ad-hoc sensor networks. In: Proceedings of IEEE INFOCOM, pp 1380-1387

8. Tian D, Georganas D (2002) A Coverage-preserving node scheduling scheme for large wireless sensor networks. In: Proceedings of ACM international workshop wireless sensor networks and applications, pp 32-41

9. Zhang H, Hou JC (2005) Maintaining sensing coverage and connectivity in large sensor networks. Int J Wirel Ad Hoc Sens Netw 1(1-2):89-124

10. Dhillon S, Chakrabarty K (2003) Sensor placement for effective coverage and surveillance in distributed sensor networks. In: IEEE wireless communications network, pp 1609-1614

11. Gonzalez-Ba HH, Latombe JC (2000) A randomized art-gallery algorithm for sensor placement. In: 16th ACM symposium computing geometry, pp 232-240 
12. Guestrin C, Krause A, Singh AP (2005) Near-optimal sensor placements in Gaussian processes. In: 22th international conference machine learning, pp 265-272

13. Latombe JC (1991) Robot motion planning. Kluwer Academic Publishers, Boston

14. Urrutia J (2000) Art gallery and illumination problems. Handbook of computational geometry. Elsevier, Amsterdam, pp 973-1027

15. Zhao F, Guibas LJ (2004) Wireless sensor networks: an information processing approach. Morgan Kaufmann, San Francisco

16. Pughat A, Sharma V (2015) A review on stochastic approach for dynamic power management in wireless sensor networks. Hum-centric Comput Inf Sci 5(4):1-14

17. Khoobjou E, Mazinan AH (2017) On hybrid intelligence-based control approach with its application to flexible robot system. Hum-centric Comput Inf Sci 7(5):1-18

18. Lee J, Chae H, Hong K (2015) A fainting condition detection system using thermal imaging cameras based object tracking algorithm. J Converg 6(3):1-15

19. Akshay N, Kumar MP, Harish B, Dhanorkar S (2010) An efficient approach for sensor deployments in wireless sensor network. In: International conference on emerging trends in robotics and communication technologies, Chennai, 3-5 Dec 2010, pp 350-355

20. Muhamad WNW, Rodzi NH, Isa NM, Ab Wahab N (2010) Efficient network coverage for wireless sensor networks. In: TENCON 2010-2010 IEEE region 10 conference, pp 240-244

21. Takahara G, Xu K, Hassanein H (2007) Efficient coverage planning for grid-based wireless sensor networks. In: IEEE international conference on communications [ICC], Glasgow, Scotland, 24-28 June 2007, pp 3522-3526

22. Ma C, Shi H, Yan G, LiY (2010) Deployment strategy with constraints of coverage and connectivity in wireless sensor networks. In: 12th IEEE international conference on communication technology (ICCT). Nanjing, 11-14 Nov 2010, pp 365-368

23. Wang J, Medidi S (2008) Mesh-based coverage for wireless sensor networks. In: IEEE global telecommunications conference, New Orleans, LO, Nov 30-Dec 4 2008, pp 1-5

24. Xu W, Hao X, Dang C (2008) Connectivity probability based on star type deployment strategy for wireless sensor networks. In: 7th world congress on intelligent control and automation, Chongqing, China, 25-27 June 2008, pp 1738-1742

25. Azlina NAA, Aziz KA, Ismail WZW (2009) Coverage strategies for wireless sensor networks. World Acad Sci Eng Technol 3(2):135-140

26. Khazan N, Motlagh ABNG (2012) Node placement and coverage in asymmetrical area. Int J Adv Res Comput Sci Softw Eng 2(11):278-282

27. Lee JH, Kim K, Lee SC, Shin BS (2014) An efficient localization method based on adaptive optimal sensor placement. Int J Distrib Sens Netw 2014:1-11, Article ID 983618

28. Manolakis DE (1996) Efficient solution and performance analysis of 3-D position estimation by trilateration. IEEE Trans Aerosp Electronic Syst 32(4):1239-1248

29. Bulut A, Shin P, Yan L (2005) Real-time nondestructive structural health monitoring using support vector machines and wavelets. In: Proceeding of the conference on advanced sensor technologies for nondestructive evaluation and structural health monitoring (NDE'05), San Diego, CA, USA, March 2005

30. Cortes C, Vapnik V (1995) Support-vector networks. Mach Learn 20(3):273-297

31. Agrawal R, Srikan R (1994) Fast algorithms for mining association rules in large databases. In: Proceedings of the 20th international conference on very large data bases, VLDB, Santiago, Chile, September 1994, pp 487-499

32. Han J, Pei J, Yin Y (2000) Mining frequent patterns without candidate generation. In: Proceedings of 2000 ACM SIGMOD international conference management of data (SIGMOD'00), Dallas, TX, pp 1-12

33. Wu Y, lanakiev K, Govindaraju V (2002) Improved k-nearest neighbor classification. Pattern Recogn 35(10):2311-2318

\section{Submit your manuscript to a SpringerOpen ${ }^{\circ}$ journal and benefit from:}

- Convenient online submission

- Rigorous peer review

- Open access: articles freely available online

- High visibility within the field

- Retaining the copyright to your article

Submit your next manuscript at $\gg$ springeropen.com 